\title{
Rapid, Label-free Pathogen Identification System for Multidrug-Resistant Bacterial Wound Infection Detection on Military Members in the Battlefield
}

Y. Chen ( $\nabla$ mechen@optowares.com )

Optowares, Inc

J. Chau

Optowares, Inc

J. Yoon

Optowares, Inc

J. Hladky

Optowares, Inc

Research Article

Keywords: Multidrug resistance bacteria, deployable, wound infection, label-free, antimicrobial susceptibility, ATR-FTIR

Posted Date: September 7th, 2021

DOl: https://doi.org/10.21203/rs.3.rs-865537/v1

License: (c) (i) This work is licensed under a Creative Commons Attribution 4.0 International License. Read Full License 


\section{Abstract}

US military service members experiencing combat-related wounds have higher risk of infection by multidrug-resistant bacteria. The gold standard culture-based antimicrobial susceptibility testing (AST) is not feasible in the battlefield environment. Thus, a rapid deployable system for bacteria identification and AST directly from wound sample is urgently needed. We report a Rapid, Label-free Pathogen Identification (RAPID) diagnostic system based on ATR-FTIR method to detect and distinguish multi-drug resistant strains for six different species in the ESKAPEE group. Our RAPID system combines on-broad sample processing to isolate and enrich bacteria cells from wound sample, ATR-FTIR measurement to detect antimicrobial-induced bacterial cell spectral changes, and machine learning model for automated, objective, and quantitative spectral analysis and unknown sample classification. Based on experimental results, our RAPID system is a promising technology for label-free, sensitive $\left(10^{4} \mathrm{cfu} / \mathrm{mL}\right.$ from mixture), species-specific (>95\% accuracy), rapid (<10 min for identification, 4 hours for AST) bacteria detection directly from wound samples.

\section{Introduction}

US military service members who are evacuated from the battlefield due to injuries had likely sustained traumatic insults such as explosions, gunshot wounds and vehicle accidents. They have higher chance of developing infection while waiting for transfer and after admission to military medical care facilities due to extensive skin and soft tissue injuries [ , , ]. Studies had reported that wound infections occur in $17 \%$ $25 \%$ of combat-related injuries in the US military treatment facilities. [ , ]. Among these infections, $14 \%-$ $22 \%$ are found to be caused by multidrug-resistant (MDR) bacteria [, , ]. MDR infections in combat wounds could lead to higher amputation rate, more subsequent operations, greater risk for developing chronic infection, osteomyelitis and sepsis, and readmission or extended stay in the medical facility [10, ]. Furthermore, treatments of MDR infections on combat-related wounds requires high medical resource consumption and are reportedly less successful and prolonged compared to civilian wounds []. Therefore as preventive measures, the patient is often given broad-spectrum antimicrobial treatment upon arrival to the medical facilities, without knowing the pathogen identity or the antimicrobial susceptibility profiles [].

Global emergence of MDR pathogens is threatening the availability and effectiveness of antimicrobials [, , ,]. As the trend of drug resistance emerging at faster pace than new FDA-approved antimicrobials is unlikely to be reversed in the foreseeable future [ ], it is paramount to preserve the effectiveness of currently available antimicrobials. Early diagnosis of the pathogen is the key for allowing conservative and effective use of antimicrobials $[,,$,$] .$

Unfortunately, there is currently no FDA-cleared, label-free, deployable, soldier-friendly, stable (no coldstorage requirement) and rapid (<30 minutes) for bacteria identification and AST in the field environment. The gold standard culture-based antimicrobial susceptibility testing (AST) is not feasible in the battlefield. Culture-based AST needs to be conducted under aseptic condition in the laboratories by specialists and uses reagents that requires cold storages, which may not be workable due to logistic and 
manpower constrains in an austere battlefield environment. Furthermore, the turnaround time for the culture-based AST is slow ( days) and thus not feasible for routine sampling to monitor patient progress.

Molecular method such polymerase chain reaction (PCR) is a promising alternative to the culture-based AST. PCR screens for the presence of known genes that confer antimicrobial resistance [ ]. However, the high sensitivity and specificity of PCR makes it prone to false positive and false negative due to sample cross-contamination []. The sample-to-answer time for PCR-based method is 2-8 hours for species identification and $>12$ hours for antimicrobial susceptibility test with automated instrumentation [. More importantly, PCR-based method is unable to detect all antimicrobial resistance because many phenotypically resistant strains exhibit resistances due to a combination of mechanisms such as enhanced beta-lactamase production, membrane impermeability due to loss of porins and expression of efflux pumps on their cell membrane $[,$, , .

Our Rapid Label-free Pathogen Identification (RAPID) system based on ATR-FTIR (Fourier-transformed Infrared Spectroscopy with Attenuated Total Reflection modality) technique has the potential to fulfil the need for sensitive and specific detection of MDR bacteria strains in the field environment. Combining with our proprietary sample processing method to enrich intact bacterial cells from wound samples and our machine-learning based analysis software package, our system can detect the presence of bacterial cells in less than 10 minutes and distinguish MDR strains in about 4 hours in the field environment and distinguish MDR strains at as low as $10^{4} \mathrm{cfu} / \mathrm{mL}$ concentration from wound sample.

FTIR is a versatile analytical technique for label-free micro-organism detection and identification [ ]. FTIR spectrum of a given molecule is highly specific due to the dense chemical information contained in a single spectrum. FTIR is a sensitive method for analyzing real-world bacterial samples, with a detection limit between $10^{3}-10^{5} \mathrm{cfu} / \mathrm{mL}$ [ ], which is several orders of magnitude lower than average bacteria concentration found in the pus drainage from infected wound $\left(10^{7}\right.$ to $\left.10^{8} \mathrm{cfu} / \mathrm{mL}\right)[$, ]. However, the range of concentration and impurities FTIR will tolerate are narrow []. The presence of bulk water is extremely problematic for FTIR transmission measurements. Thus, samples need to be prepared into a thin, dried pellet through tedious sample processing steps.

Attenuated total reflection (ATR) was invented as a sampling technique to bring FTIR to a wider variety of samples with various contaminants and optical densities. In ATR the infrared light propagates within an ATR element via total internal reflection. Since the IR radiation is not transmitted through the bulk of the sample, the sample does not need to be prepared as a thin pellet. The limited depth of interaction (1-2 $\mu \mathrm{m}$ ) of the IR radiation beyond the ATR crystal surface makes possible for collecting high-quality spectra in the presence of bulk water, while remain sufficiently deep to cover a monolayer of bacteria on the crystal surface. Moreover, most modern ATR instruments are capable for acquiring time-dependent spectra by repeatedly interrogating the ATR system and with software design to track intensity changes over time, giving ATR the ability for continuous monitoring for chemical/biological activities of the bacteria. 
Many examples of how the ATR sampling technique enables micro-organisms detection by FTIR in complex aqueous environment had been reported in literature. For example, ATR-FTIR was used to study swarming behavior of bacterial cells [, to monitor growth and cyclic changes in bacterial biofilm population [ , ], and to study penetration of antimicrobial agents into the bacterial biofilm [ ].

In this report we describe the capability of our ATR-FTIR based system to detect and distinguish six different MDR and six antimicrobial-susceptible bacteria strains from spiked samples under laboratory environment. We demonstrate the strain-specificity, sensitivity and selectivity of our ATR system in recognizing unique bacterial spectral features in pure solution and in complex mixture through quantitative spectral analysis, and the use of machine learning models to automate the analysis process.

\section{Results And Discussion}

Specificity: Utilize Antimicrobial-induced Bacterial Cell Spectral Changes to Distinguish MDR and Susceptible Strains.

We obtained robust and reproducible ATR spectra (Figure S1) of dried cell pellet at $10^{9}-10^{12} \mathrm{cfu} / \mathrm{mL}$ before and after antimicrobials incubation. $10^{9}-10^{12} \mathrm{cfu} / \mathrm{mL}$ was used because the size of the cell pellet at this concentration range could be readily obtained by centrifugation and handled by pelleting in our laboratory. These spiked bacterial samples were prepared in $5 \%$ dextrose/saline, to allow us to obtain high-resolution ATR spectra and study the bacterial spectral feature with minimal interference from the solvent and other contaminants in the sample. The high-quality ATR spectra for all 12 strains are shown in Fig. 1 .

We then compared our results with literature to understand the assignment of functional groups associated with major peaks in the bacterial spectrum. Our ATR bacterial spectra from $4000-1500 \mathrm{~cm}^{-1}$ are consistent compared to literature $[, \ldots, \ldots]$. Most of these peaks are vibrational bands of protein and fatty acid, likely part of the cell wall components due to their proximity to the ATR crystal surface. This means that achieving strain specificity with ATR technology is possible since each strain will likely have unique membrane protein and fatty acid compositions. It also means that changes to bacterial cell membrane, such as those induced by interaction with antimicrobial, would be detectable by ATR.

In Fig. 1 we observed peak shifts and peak intensity changes common to all 12 strains, in two spectral regions as highlighted by the red and blue rectangular boxes. Figure 2 shows a zoom-in view of these two regions. These spectra were analyzed quantitatively to find any common trends exist among the MDR strains that can be used to distinguished from the susceptible ones, and correlate to the number of viable cells in sample. The following definitions were used during quantitative analysis.

1. $A U C=$ area under the curve. $\% A U C$ reduction $=\left(A U C_{M D R}-A U C_{\text {susceptible }}\right) /\left(A U C_{M D R}\right) \times 100 \%$.

2. $\mathrm{CFU}=$ colony forming unit. $\%{ }_{\log } \mathrm{CFU}$ reduction $=\left(\log \mathrm{CFU}_{\text {before }}-\log \mathrm{CFU} \mathrm{U}_{\text {after }}\right) /\left(\log \mathrm{CFU}_{\text {before }}\right) \times 100 \%$. 
The quantitative spectral analysis results are shown in the table at the bottom of Fig. 2. Quantitative spectral analysis of peaks in Fig. 2 shows (1) susceptible strain have larger \% AUC reduction than the MDR one in all six bacterial species in both spectral regions, and (2) \%AUC reduction can be correlated to the number of viable cells as measured by $\%{ }_{\text {log }} \mathrm{CFU}$ reduction. Larger \% AUC reduction corresponds to larger \% ${ }_{\log } \mathrm{CFU}$ reduction and vice versa. We verified that these peak intensity changes were entirely due to change on the bacteria cell instead of spectroscopic contributions from the added antimicrobial solutions as shown in Figure $\mathrm{S} 2$.

Figure 1 and Fig. 2 shows that our ATR method can detect changes due to antimicrobial-induced bacterial cell changes spectroscopically and uses these changes to distinguish MDR and susceptible strains.

\section{Selectivity: Detect Bacterial Spectral Features in Mixtures}

Combat-related wounds are often contaminated by foreign materials at the point of injury (for example, dust and soil, cloth fiber from bandage and bed linen, metal pieces, and so on) [7, ]. In some infections a possibility of multiple strains can be presented [4, 11,9]. Infected wound samples are typically collected in the form of swab, pus aspirates and tissue/blood biopsy samples [, and the choice of sampling technique depends on the condition of the patient, resources available and the type of wounds. Therefore, samples from infected wounds are highly complex mixtures, and one of the most important capability of the diagnostic system is the ability to recognize each bacterial species spectral features in the mixture.

The potential of our ATR technology in detecting bacterial features in mixtures is demonstrated below by acquiring high-quality ATR spectra from two bacteria-spiked, protein-rich mixtures.

\section{Example 1: recognize bacterial spectral features in protein- rich mixture}

Figure 3 (a) shows an average of five spectra of E. coli BAA2523 in a fetal bovine serum/water (FBS, Sigma-Aldrich $\left.{ }^{\circledR}\right)$ 1:5 (v/v) mixture. Compared to the E. coli BAA2523 spectrum in $5 \%$ dextrose/saline from Fig. 1, the bacterial features were clearly distinguishable in the protein-rich mixture, as shown in the two regions highlighted by the black boxes.

\section{Example 2: distinguish MDR and susceptible strain in the same mixture}

We obtained ATR spectra of a mixture sample consisted of $60 \%$ Klebsiella pneumoniae BAA2146 (MDR) and 40\% Klebsiella pneumoniae 13883 (susceptible), and successfully recognized both strains in the mixture. The spectra of the K. pneumoniae BAA2146 sample, the K. pneumoniae 13883 sample and the $60 / 40$ mixture showed very similar spectral features after incubation with $8 \mu \mathrm{g} / \mathrm{mL}$ ceftriaxone disodium solution for 4 hours at $35^{\circ} \mathrm{C}$. When zoom-in to the $1500-1850 \mathrm{~cm}^{-1}$ region (where Amide I and Amide II vibrational features are), all three samples show different relative ratio of the $\sim 1660$ and $\sim 1630 \mathrm{~cm}^{-1}$ 
peaks marked by the green and the black arrows, respectively, as shown in Fig. 3 (b1). The mixture spectrum shows clear contribution from both strains. Quantitative analysis on the mixture spectrum reveals more contribution from K. pneumoniae BAA2146 and less from K. pneumoniae 13883, characterized by the similarity between the experimental mixture spectrum and the mathematical addition of $60 \%$ K. pneumoniae BAA2146 spectrum $+40 \%$ K. pneumoniae 13883 spectrum, as shown in Fig. 3 (b2).

In summary, Fig. 3 shows the selectivity of our ATR system to recognize high-resolution, strain-specific bacterial spectral feature in complex mixture, and use these features to distinguish MDR and antimicrobial-susceptible strains.

\section{Sensitivity: Observe Antimicrobial-induced Bacterial Cell Spectral Changes at $10^{4} \mathrm{cfu} / \mathrm{mL}$}

Figure 1-3 demonstrated the capability of our ATR system to recognize antimicrobial-induced spectral changes and discern MDR and antimicrobial-susceptible strains in cell pellet samples $\left(10^{9}-10^{12} \mathrm{cfu} / \mathrm{mL}\right)$. In this section, we demonstrate similar capability of our ATR system at much lower cell concentration of $10^{4} \mathrm{cfu} / \mathrm{mL}$.

Experimentally, two identical $50 \mathrm{~mL}$ samples of E. coli 25922 at $\sim 10^{4} \mathrm{cfu} / \mathrm{mL}$ in $0.85 \mathrm{wt} \% \mathrm{NaCl}$ solution were prepared. The bacteria cells were collected by filtering the solution through a $0.22 \mu \mathrm{m}$ PTFE membrane filter using a $47 \mathrm{~mm}$ glass vacuum filtration assembly. The membrane filter was air-dried and ATR spectra of the cells on the membrane were acquired by pressing the membrane filter against the ATR element with a pressure-clamp system.

Ertapenem sodium was added to one of the two samples to make the final concentration of $10 \mu \mathrm{g} / \mathrm{mL}$, and the sample was set for 4 hours at $35^{\circ} \mathrm{C}$ with agitation. Spectra were acquired after the filtration of the sample. For the other sample, filtration and spectral acquisition were done immediately after preparation.

The ATR spectra before and after antimicrobial incubation with this filtration + ATR procedure is compared in Fig. 4 (left). Due to the size of the filtration assembly (Fig. 4 right, $47 \mathrm{~mm}$ diameter), $50 \mathrm{~mL}$ sample volume was needed. With $10^{4} \mathrm{cfu} / \mathrm{mL}$ bacteria cell concentration, we expected that only a small fraction of the PTFE membrane will be covered by the bacterial cells. Therefore, some of the PTFE spectral features (marked by the black arrows) are observed along with the bacterial features (marked by the green arrows). However, the bacterial features, as well as the antimicrobial-induced peak intensities reduction are clearly distinguishable from the PTFE features.

In summary, Fig. 4 shows that we can observe ertapenem-induced peak changes at $10^{4} \mathrm{cfu} / \mathrm{mL}$ for a $E$. coli 25922 sample with our ATR system.

\section{Automation: Machine Learning for Objective, Quantitative Spectral Analysis and Classification}


We built a sum vector machine - discriminant analysis (SVMDA) model using the ATR spectra reported in Fig. 1. The five ATR spectra from the MDR and the susceptible strains after antimicrobial incubation in each species are combined to form one class. The results are shown in Fig. 5(a). Each dot in the figure represents one spectrum. The class membership ID predicted by the model is shown in the y-axis, and the actual class membership (i.e. the "correct" answer) is shown in the legend. Each species is color-coded.

Our SVMDA model achieved $>95 \%$ averaged analytical sensitivity and specificity for the six bacterial species. To test the performance of our SVMDA model, we applied ATR spectra from four different samples, including the ones shown in Fig. 3 , and recorded the species identity classified by the model. The results are summarized numerically in Fig. 5(b). Every spectrum in these four unknown samples were correctly classified by the model.

\section{Conclusion}

In conclusion, we demonstrated the potential of our RAPID system based on FTIR-ATR to detect and distinguish MDR strains in pure solution and in mixture, by recognizing unique bacterial ATR spectral features and antimicrobial-induced changes of these features. We demonstrated a $10^{4} \mathrm{cfu} / \mathrm{mL}$ detection limit and a machine learning model with $>95 \%$ averaged analytical sensitivity and specificity, as well as $100 \%$ accuracy in classifying species identity in four unknown samples. Our results clearly show our RAPID system ATR technology can meet the critical need for a rapid portable system suitable for front line users to detect the presence of MDR bacteria in infected combat-related wounds.

\section{Materials And Methods}

\section{MDR and Antimicrobial-Susceptible Bacterial Strain Procurement:}

Six MDR and six antimicrobial-susceptible strains were selected for this study. These 12 strains represented six different species from the ESKAPEE group - the acronym for a group of seven MDR species on World Health Organization Critical Priority I and II Pathogens lists: Staphylococcus aureus, Klebsiella pneumoniae, Acinetobacter baumannii, Pseudomonas aeruginosa, Enterobacter cloacae and Escherichia coli. The strain ID and susceptibility profile to six antimicrobials (one antimicrobial per species) are summarized in the table in Figure 1. All 12 strains were obtained from ATCC. These strains were cultured, harvested and stored according to ATCC guideline in our BSL-2 facility. We used the antimicrobial susceptibility profile and $\mathrm{MIC}_{50}$ values reported by ATCC, from the CLSI-M100 tables and literature for the strain without further verification. Bacterial samples emanated from the virgin ATCC samples were used in all the experiments (i.e. subcultured sample were not used).

Sterile, disposable, untreated cell culture flasks with $0.22 \mu \mathrm{m}$ filter cap (Culture Area: $75 \mathrm{~cm}^{2}$, VWR®) with 60-75 mL broth were used to rehydrate the sample. Each stain was emanated for 18-24 hours with constant agitation in tryptic soy broth at $37^{\circ} \mathrm{C}$, except for Klebsiella pneumoniae 13883 which required nutrient broth. After incubation, the bacterial cells were harvest by transferring the flask content to 
multiple sterile, $50 \mathrm{~mL}$ conical tubes and centrifuge at $8000 \mathrm{rpm}$ for 10 minutes to obtain cell pellet. The cell pellet is aliquoted to multiple $1.5 \mathrm{~mL}$ cryogenic vials with $20 \%$ skim milk as cryoprotectant. The vials were stored in the vapor phase of a liquid nitrogen Dewar.

Before centrifugation, $1 \mathrm{~mL}$ was sampled from the flask to determine the number of viable cells by quantitative cell culture. The procedure is following. The approximate cell concentration in the sample was estimated based on the cloudiness. Based on the estimation the $1 \mathrm{~mL}$ sample was serially diluted with tryptic soy broth or nutrient broth until reaching $10^{-10}$ to $10^{-12}$ (undiluted sample $=10^{0}$ ) level. Minimally 5 most diluted sample in the serial dilution were plated. The averaged number from these five plates was reported as the number of viable cells in the sample, typically in the $10^{11}-10^{13} \mathrm{cfu} / \mathrm{mL}$ range.

\section{ATR-FTIR Measurement Setup:}

We used a Nicolet ${ }^{\circledR}$ iS10 FTIR spectrometer (ThermoFisher ${ }^{\circledR}$ ) with a horizontal ATR accessory (HATR, PIKE Technologies $\AA$ ) to obtain all ATR spectra in this report. The enclosure of the HATR accessory was removed to allow greater access to the ATR element. Both the HATR accessory and the FTIR spectrometer were under constant nitrogen purging to minimize absorption due to atmospheric water and $\mathrm{CO}_{2}$. Both HATR accessory and the ATR element are disinfected by $70 \%$ ethanol/water between each measurement.

A multi-bounce silicon $45^{\circ}$ trapezoidal ATR element ( $80 \times 10 \times 2 \mathrm{~mm} \mathrm{~L} \times \mathrm{W} \times \mathrm{H}$, PIKE Technologies $\AA$ ) was used in all ATR measurements. We selected Si because of its hardness and chemical resistance to $70 \%$ ethanol/water compared to other ATR element materials. An air background spectrum was collected before each ATR measurement. The bacterial ATR spectra were acquired and analyzed using OMNIC® Software (ThermoFisher ${ }^{\circledR}$ ) with the following setting:

Spectral region recorded: $4000-1500 \mathrm{~cm}^{-1}$. Spectral Resolution: $4 \mathrm{~cm}^{-1}$. Atmospheric suppression: On. \# of scans: 256.

\section{Bacterial Sample Preparation:}

An $0.25 \mathrm{~mL}$ aliquot sample was removed from the liquid nitrogen storage and thawed at $37^{\circ} \mathrm{C}$. The content was transferred to two $0.5 \mathrm{~mL}$ microcentrifuge tubes, one would be used for acquiring spectra before antimicrobial incubation and the other one would be for after antimicrobial incubation. Cell pellet was collected by centrifuging at $10,000 \mathrm{~g}$ for 5 minutes. The skim milk supernatant was removed and 80 - $100 \mu \mathrm{L} 5 \%$ dextrose/saline (freshly prepared and double-filtered by $0.22 \mu \mathrm{m}$ filter) were used to wash the cell pellet 2-3 times.

To obtain spectra before antimicrobial incubation, immediately after the last wash the cell pellet was dispersed in $4 \mu \mathrm{L} 5 \%$ dextrose/saline. All liquid contents were transferred to the Si ATR crystal surface using $1 \mu \mathrm{L}$ pipette tips in 7-8 evenly distributed droplets along the length of the crystal. The added $4 \mu \mathrm{L}$ liquid helped to lift the cell pellet off the microcentrifuge tube wall. The total sample volume $(4 \mu \mathrm{L}+$ cell pellet) should be $8-10 \mu \mathrm{L}$. An identical Si ATR element was placed over the sample to form a 
"sandwich". The sample was smeared into a thin film of liquid with the top crystal. The smeared sample would dry up in air in 1 -2 minutes, forming a thin, uniform layer of dried bacterial cells. The ATR element was inserted into the HATR accessory and a set of five ATR spectra was acquired.

To obtain spectra after antimicrobial incubation, antimicrobial stock solution at $2 x$ concentration was added to the bacterial sample by mixing equal volume of the stock solution with the bacteria sample. The antimicrobial stock solution was prepared within 30 minutes before experiment in $5 \%$ dextrose saline and stored at $2-8{ }^{\circ} \mathrm{C}$ until use. Then the mixture was incubated at $35^{\circ} \mathrm{C}$ for 4 hours without agitation. After incubation, the cell pellet was collected, washed. ATR spectra were then acquired following the procedure described before.

\section{ATR Spectra Preprocessing, Building SVMDA models and Classification In Unknown Samples:}

Each ATR spectrum was subjected to spectral preprocessing before compiling into a spectral reference library. Spectral preprocessing can help to improve classification model sensitivity and specificity by enhancing inherent differences between classes while reducing variation within the sample class. The library was used to train classification models.

The ATR range cutoff at low wavenumber for the Si HATR element is $1500 \mathrm{~cm}^{-1}$ in Mid-IR. Thus the spectral region between $1500-1200 \mathrm{~cm}^{-1}$ was recorded as shown Figure 1, but not considered in the spectral analysis. Each ATR spectrum was baselined by an "Automatic Baseline Correct" algorithm in the OMNIC software so that spectra from different strains and from before vs. after antimicrobial incubation could be quantitatively compared.

To prepare for training classification model to distinguish the six different bacterial species, the spectra were min-max normalized to ensure the model would "focus" on finding species-specific characteristics instead of the absolute peak intensity differences within the dataset.

We utilized the PLS_Toolbox ${ }^{\circledR}$ from Eigenvector Research, Inc (Mason, MA), a chemometric toolbox on MATLAB platform, to build our classification model. We first built and optimized a partial least square discriminant analysis (PLSDA) pre-model. PLSDA is a "supervised" version of principal component analysis, i.e. class ID are requested from the user and utilized to decide class boundary. We examined the pre-model to ensure genuine differences existed between classes. Then a sum vector machine discriminant analysis (SVMDA) classification model was built based on the PLSDA results. SVMDA is a model optimization technique; the class boundary SVMDA is non-linear to allow better classification for data points that are too close to the boundary in the PLSDA pre-model. The curviness of boundary is governed by the data points closest to the boundary (i.e. "support vector"). The SVMDA model parameters were fine-tuned and compiled, and the analytical sensitivity and specificity were calculated.

To test the performance of the SVMDA model, ATR Spectra from 4 different unknown samples were acquired and baseline/normalized by methods described above. The model performance was assessed by determining the \% of data from these unknow sample that were correctly classified the model. 


\section{Declarations}

\section{Acknowledgement}

This report is based upon work supported by the DHA SBIR Program/U.S. Army Medical Research and Development Command (USAMRDC)/U.S. Air Force Material Command under Contract No. W81XWH-20P-0146.

\section{Author Contributions}

Conceived and design the experiments: Y. Chen. Perform the experiment: Y. Chen, J. Chau, J. Hladky. Analyze the data: Y. Chen. Wrote the paper: Y. Chen, J. Yoon, J. Hladky.

Corresponding author: Correspondence to Y. Chen (mechen@optowares.com).

Additional Information

Competing Interests: The authors declare no competing interest.

\section{References}

1. E.N. Johnson, T.C. Burns, R.A. Hayda, D.R. Hospenthal, C.K. Murray. Infectious complications of open type III tibial factures among combat casualties. Clin. Infect. Dis. 45, 409-415 (2007).

2. D.R. Tribble, L.R. Lewandowski, B.K. Potter, et al. Trauma Infectious Disease Outcomes Study Group. Osteomyelitis risk factos related to combat trauma open tibia fractures: a case-control analysis. $J$. Orthop. Trauma. 32, e344-e353 (2018).

3. K.V. Brown, C.K. Murray, J.C. Clasper. Infectious complications of combat-related mangled extremity injuries in the British military. J. Trauma. 69, S109-S115 (2010).

4. K.A. Davis, K.A. Moran, K. McAllister, P.J. Ray. Multidrug-resistant Acineotbacter extremity infections in soldiers. Emerg. Infect. Dis. 11, 1218-24 (2005).

5. A.C. Weintrob, M.P. Roediger, M. Barber, et al. Natural history of colonization with gram-negative multidrug-resistant organisms among hospitalized patients. Infect. Control. Hosp. Epidemiol.31, 330337 (2010).

6. O. Saeed, D.R. Tribble, K.A. Biever, H.K. Crouch, M. Kavanaugh. Infection prevention in combat-related injuries. Mil. Med. 183, 137-141 (2018).

7. T.E. Wallum, H.C. Yun, E. A. Rini, et al. Pathogens present in acute mangled extremities from Afghanistan and subsequent pathogen recovery. Mil. Med. 180, 97-103 (2015).

8. T.J. Vento, D.W. Cole, et al. Multidrug-resistant gram-negative bacteria colonization of healthy US military personnel in the US and Afghanistan. BMC Infect. Dis. 13, 68 (2013).

9. J.B. Ronat, J. Kakol, M.N. Khoury, et al. Highly drug-resistant pathogens implicated in burnassociated bacteremia in an Iraqi Burn Care Unit. PLoS ONE. 9, e101017 (2014). 
10. A. Älgå, et al. Infection With High Proportion of Multidrug-resistant Bacteria in Conflict-related Injuries is Associated with Poor Outcomes and Excess Resource BMC Infect. Dis. 18, 233-260 (2018).

11. J.H. Calhoun, C.K. Murray, M.M. Manring. Multidrug-resistant Organisms in Military Wounds from Iraq and Afghanistan. Clin. Orthop. Relat. Res. 466, 1356-1362 (2008).

12. P.G. Bowler, B. I. Buerden, and D. G. Armstrong. Wound Microbiology and Associated Approaches to Wound Management. Clin. Microbiol. Rev. 14, 244-269 (2001).

13. R.G. Frykberg and J. Banks. Challenges in the Treatment of Chronic Wounds. Adv. Wound Care (New Rochelle). 4, 560-582 (2015).

14. Z.T. Sahli, et al. Microbiology and Risk Factors Associated with War-related Wound Infections in the Middle East. Epidemiol. Infect. 144, 2848-2857 (2016).

15. A. Merens, et al. Prevention of Combat-related Infections: Antimicrobial Therapy in Battlefield and Barrier Measures in French Military Medical Treatment Facilities. Travel. Med. Infect. Dis. 12, 318-329 (2014).

16. Center for Disease Dynamics, Economics \& Policy. 2015. State of the World's Antibiotics, 2015. CDDEP: D.C.

17. Antibiotic Resistance Threats in the United States, U.S. Department of Health and Human Services, CDC; 2019.

18. O'Neill J (2014) Antibiotic resistance: Tackling a crisis for the health and wealth of nations. The Review on Antibiotic Resistance (HM Government, London).

19. Antibiotic resistance: promoting critically needed antibiotic research and development and appropriate use ("stewardship") of these precious drugs. Testimony of the Infectious Diseases Society of America (IDSA) before the House Committee on Energy and Commerce Subcommittee on Health, June 9, 2010. Accessed February 19, 2020.

20. C.L. Ventola. The antibiotic resistance crisis: part 1: causes and threats, P. T. 40, 277-283 (2015).

21. I.N. Okeke, et al. Diagnostics as essential tools for containing antibacterial resistance. Drug Resist. Updat. 14, 95-106 (2011).

22. C.K. Murray, et al. Bacteriology of War Wounds at The Time of Injury. Mil. Med. 171, 826-829 (2006).

23. C.K. Murray. Field Wound Care: Prophylactic Antibiotics. Wilderness Environ. Med. 28, S90-S102 (2017).

24. O. Saeed, et al. Infection Prevention in Combat-Related Injuries. Mil. Med. 183, 137-141 (2018).

25. S.A. Cunningham, T. Noorie, D. Meunier, N. Woodford, R. Patel. Rapid and Simultaneous Detection of Genes Encoding Klebsiella pneumonia Carbapenemase (blaKPC) and New Delhi Metallo- $\beta$ Lactamase (blaNDM) in Gram-negative Bacilli, J. Clinical Micrbiology 51, 1269 (2013).

26. M. Hindiyeh, et al. Rapid Detection of blaKPC Carbapenemase Genes by Internally Controlled RealTime PCR Assay Using Bactec Blood Culture Bottles. J. Clin. Microbiol. 49, 2480-2484 (2011).

27. J.J. Mauer. Rapid Detection and Limitations of Molecular Techniques. Annu. Rev. Food Sci. Technol. 2, 259-279 (2011). 
28. ViTek 2 Product safety "https://www.biomerieux-usa.com/industry/vitek-2-product-safety". Accessed August 2021.

29. G. Kapoor, S. Saigal, A. Elongavan. Action and resistance mechanisms of antimicrobials: a guide for clinicians. J. Anaesthesiol. Clin. Pharmacol. 33, 300-305 (2017).

30. H. Zou, et al. CP-CRE/non-CP-CRE Stratification and CRE Resistance Mechanism Determination Help In Better Managing CRE Bacteremia Using Ceftazidime and Aztreonam-Avibactam, Infect. Drug. Resist. 12, 3017-3027 (2019).

31. J. Iredell, et al. Antibiotic Resistance in Enterobacteriaceae: Mechanisms and Clinical Implications. BMJ. 2016 Feb 8; 352:h6420.

32. R. Bouganim, et al. The Clinical and Molecular Epidemiology of Noncarbapenemase-Producing Carbapenem-Resistant Enterobacteriaceae. A Case-Case-Control Matched Analysis. Open Forum Infec. Dis. 7, ofaa299 (2020).

33. D. Helm, et al. Classification and identification of bacteria by Fourier-transform infrared spectroscopy. J. Gen. Microbiol. 137, 69-79 (1991).

34. W. Bouhedja, et al. ATR-FTIR spectroscopic investigation of E. coli transeonjugants beta-lactamsresistance phenotype. FEBS Lett. 412, 39-42 (1997).

35. R. Davis, J. Irudayaraj, B.L. Reuhs, L.J. Mauer. Detection of E. coli 0157:H7 from ground beef using Fourier transform infrared (FT-IR) spectroscopy and chemometrics. J. Food Sci. 75, M340-346 (2010).

36. N. Nicolaou, R. Goodacre. Rapid and quantitative detection of the microbial spoilage in milk using Fourier transform infrared spectroscopy and chemometrics. Analyst 133, 1424-1431 (2008).

37. M.A. Lewis, et al. Quantitative bacteriology of acute dento-alveolar abscesses. J. Med. Microbio1. 21, 101-104 (1986).

38. P. Borer, et al. ATR-FTIR spectroscopic study of the adsorption of desferrioxamine $B$ and aerobactin to the surface of lepidocrocite ( $\mathrm{\gamma}-\mathrm{FeOOH})$. Geochim. Cosmochim. Acta 73, 4661-4672 (2009).

39. P.R. Brejna, P.R. Griffiths. Electroless deposition of silver onto silicon as a method of preparation of reproducible surface-enhanced Raman spectroscopy substrates and tip-enhanced Raman spectroscopy tips. Appl. Spectrosc. 64, 493-499 (2010).

40. M. Gué, et al. Bacteria swarming: a biochemical time-resolved FTIR-ATR study of Proteus mirabilis swarm-cell differentiation. Biochemistry 40, 11938-11945 (2001).

41. F. Quilès, F. Humbert, A. Delille. Analysis of changes in attenuated total reflection FTIR fingerprints of Pseudomonas fluorescens from planktonic state to nascent biofilm state. Spectrochimica. Acta. Part A, Molecular and biomolecular spectroscopy 75, 610-616 (2010).

42. J. Pink, et al. An FTIR study of Pseudomonas aeruginosa PAO1 biofilm development: interpretation of ATR-FTIR data in the $1500-1180 \mathrm{~cm}^{-1}$ region. Biofilms. 2, 165-175 (2005).

43. P. Stenclova, et al. Cyclic changes in the amide bands within Escherichia coli biofilms monitored using real-time infrared attenuated total reflection spectroscopy (IR-ATR). Appl. Spectrosc. 73, 424- 
432 (2019).

44. P.A. Suci, J.D. Vrany, M.W. Mittelman, Investigation of interactions between antimicrobial agents and bacterial biofilms using attenuated total reflection Fourier transform infrared spectroscopy, Biomaterials 19, 327-339 (1998).

45. P.A. Suci, M.W. Mittelman, F.P. Yu, G.G. Geesey. Investigation of ciprofloxacin penetration into Pseudomonas aeruginosa biofilms. Antimicrob. Agents Chemother. 38, 2125-2133 (1994).

46. L.E. Rodriguez-Saona et al. Detection and Identification of Bacteria in a Juice Matrix with Fourier Transform-Near Infrared Spectroscopy and Multivariate Analysis, J. Food Prot., 67, 2555-2559 (2004).

47. M.M. Mossoba et al. Application of a Novel Hydrophilic Infrared-Transparent Membrane to the Differentiation between Microcolonies of Enterobacter sakazakii and Klebsiella pneumoniae, J. Food Prot. 70, 1241-1245 (2007).

48. R. Davis, et al. Differentiation of Live, Dead and Treated Cells of Escherichia coli 0154:H7 using FT-IR Spectroscopy, J. Appl. Microbiol. 112, 743-751 (2011).

49. M. Al-Holy et al. The Use of Fourier Transform Infrared Spectroscopy to Differentiate Escherichia coli 0157:H7 from Other Bacteria Inoculated into Apple Juice, Food. Microbiol., 23, 162-168 (2006).

50. X. Lu et al. Investigating Antibacterial Effects of Garlic (Allium sativum) Concentrate and GarlicDerived Organosulfur Compounds on Campylobacter jejuni by Using Fourier Transform Infrared Spectroscopy, Raman Spectroscopy and Electron Microscopy, Appl. Environ. Microbiol. 77, 52575269 (2011).

51. R. Davis and L.J. Mauer. Fourier Transform Infrared (FT-IR) Spectroscopy: A Rapid Tool for Detection and Analysis of Foodborne Pathogenic Bacteria. Current Research, Technology and Education Topics in Applied Microbial Biotechnology: A. Mendez-Vilas (Ed.) (2010).

52. W.G.P. Eardley, et al. Infection in conflict wounded. Philos. Trans. R. Soc. Lond. B. Biol. Sci., 366, 204218 (2011).

53. L.R. Copeland-Halperin, et al. Sample Procurement for Cultures of Infected Wounds: A Systematic Review. J. Wound Care, 25, S4-6, S8-10 (2016).

\section{Figures}




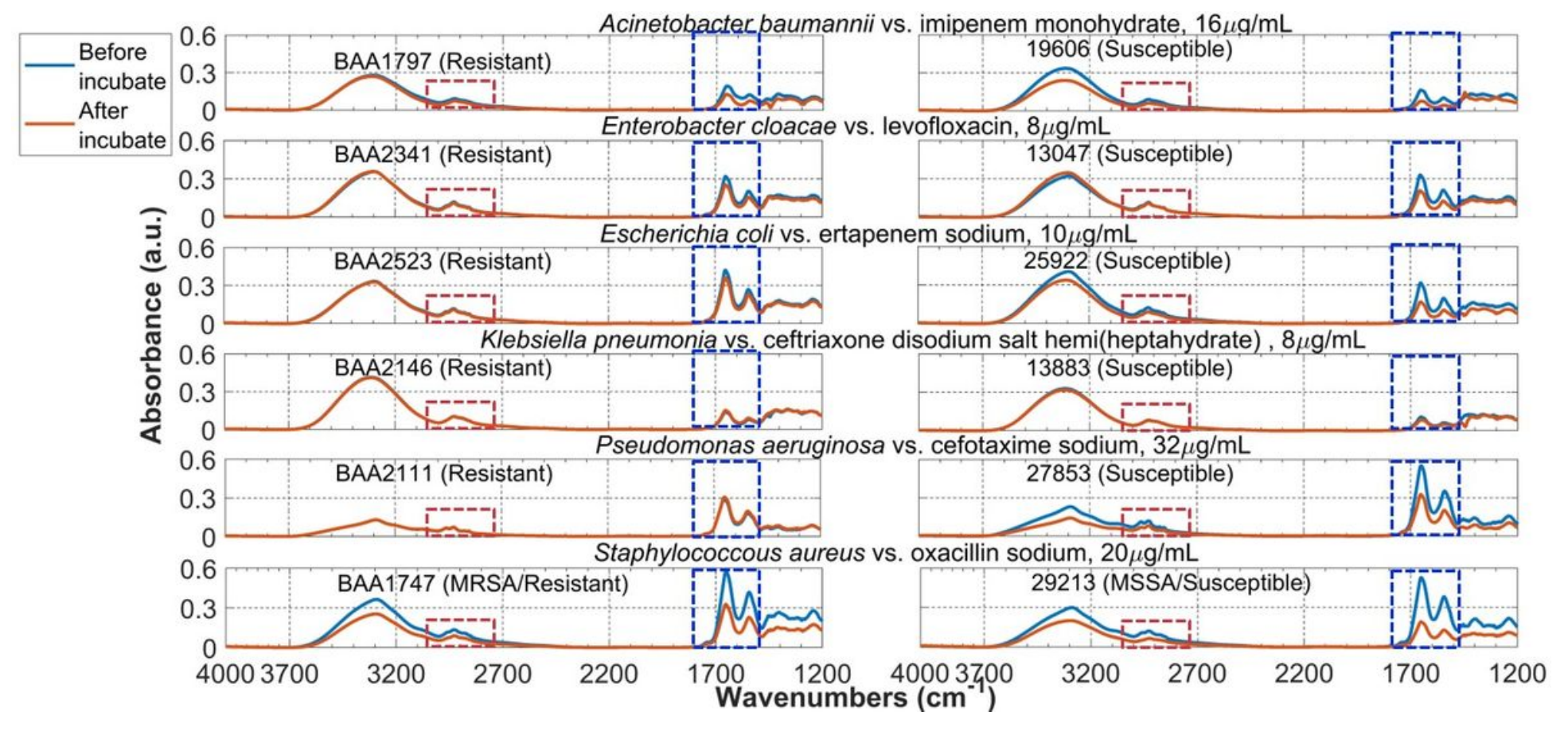

Figure 1

Specificity of the ATR system. Compare averaged ATR spectra of the bacteria cell before and after incubation with antimicrobial solution at $35^{\circ} \mathrm{C}$ for 4 hours. Consistent spectral changes are observed in two highlighted spectra regions. 


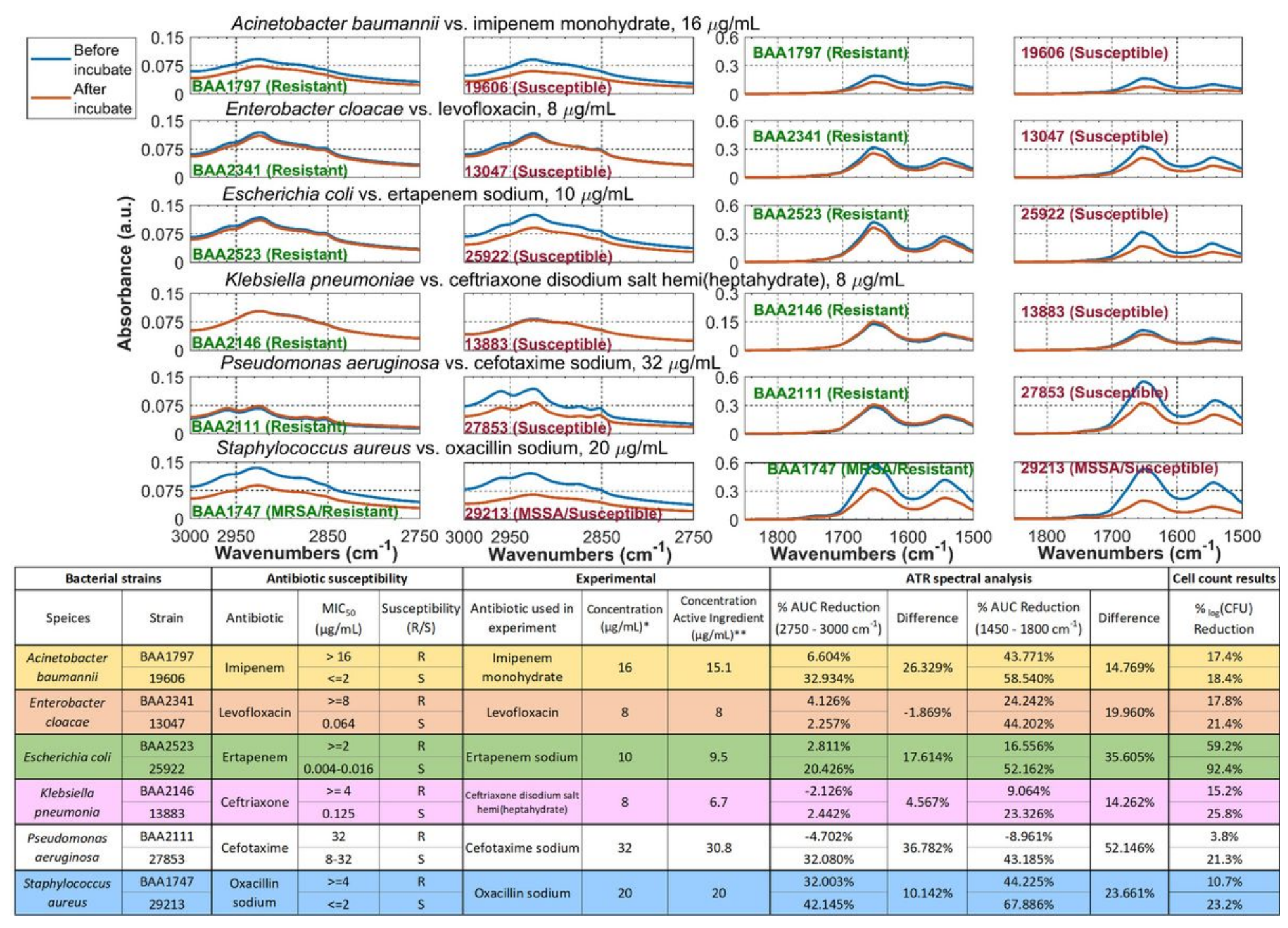

Figure 2

(Top) Zoom-in view to the two highlighted regions in each strain from Figure 1 to show the common spectral change trend between MDR and antimicrobial-susceptible strains in six species. (Bottom) Table summary (1) Strain designation, antimicrobial susceptibility to six different antimicrobials, MIC50 values and the sources for the MIC50 values. (2) Reagent used in experiment and the concentration of active ingredient. * All antimicrobial solution is prepared in $5 \%$ dextrose saline. ${ }^{*}$ Estimated concentration of antimicrobial in the prepared solution. (3) Area Under the Curve (AUC) calculation for each spectrum. (4) \# of viable colonies determined by quantitative overnight culture for each strain, before and after antimicrobial incubation. 

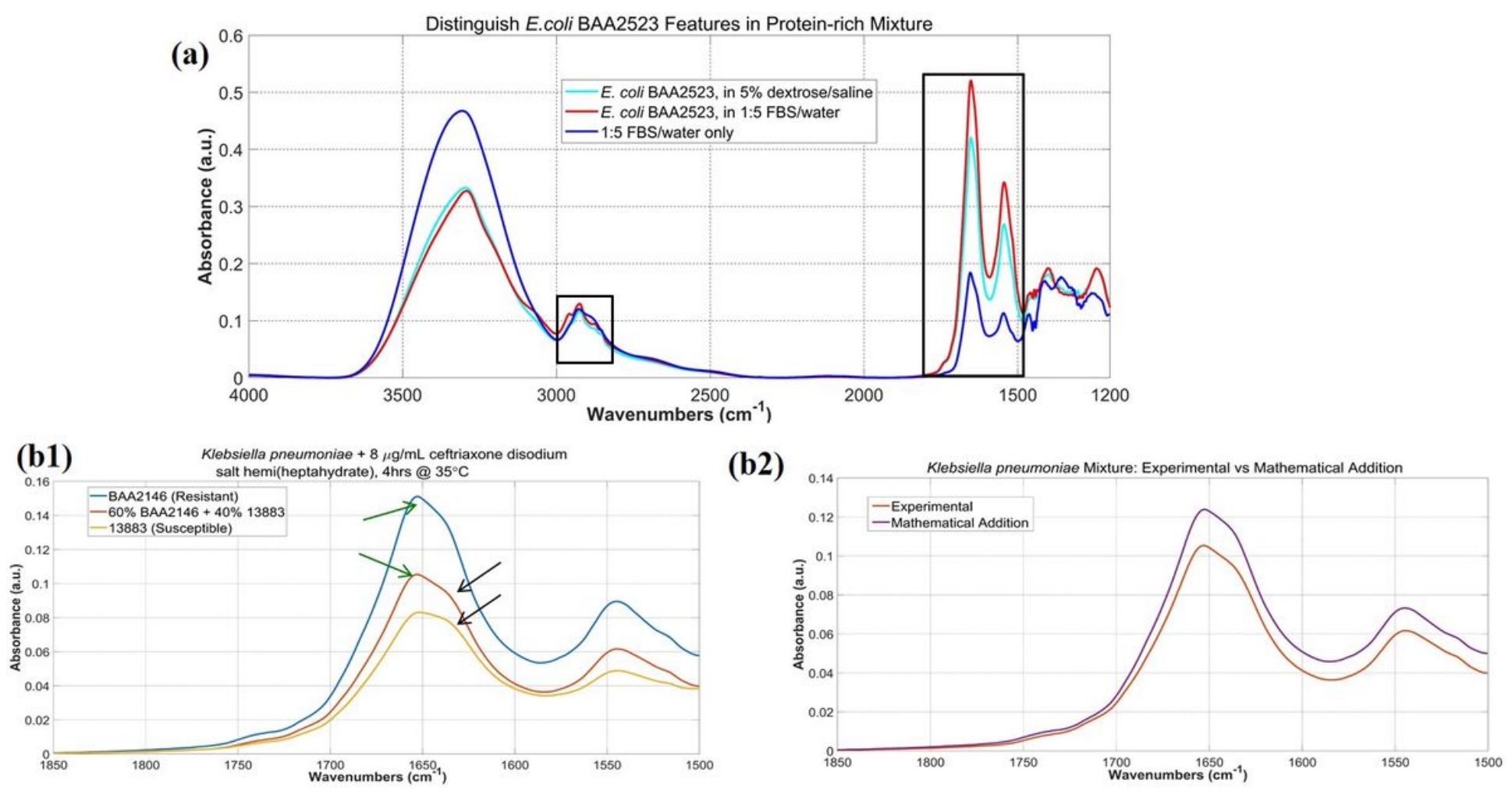

\section{Figure 3}

Selectivity of the ATR system. (a) averaged ATR spectra of E. coli BAA2523 in (cyan) 5\% dextrose saline and in (red) FBS/water 1:5 mixture. The spectra of the FBS/water 1:5 mixture itself is shown in Blue. The two black rectangular boxes highlight two spectral regions where clear spectral differences can be seen. (b1) ATR spectra of K. pneumoniae BAA2146 (MDR), K. pneumoniae 13883 (susceptible) and a mixture of $60 \%$ BAA2146 and $40 \% 13883$. Zoom-in view to the Amide I - Amide II spectral region (1500 - 1850 $\mathrm{cm}-1$ ). \# of viable cells in all three are in the $107 \mathrm{cfu} / \mathrm{mL}$ range. (b2) Contributions from BAA2146 and 13883 are clear in the mixture spectrum, marked by similarity between the experimental spectrum and the mathematical addition of the BAA2146 and 13883.

Detect Antibiotic-Induced Bacterial Spectral Changes @ $10^{4} \mathrm{cfu} / \mathrm{mL}$ LOD,

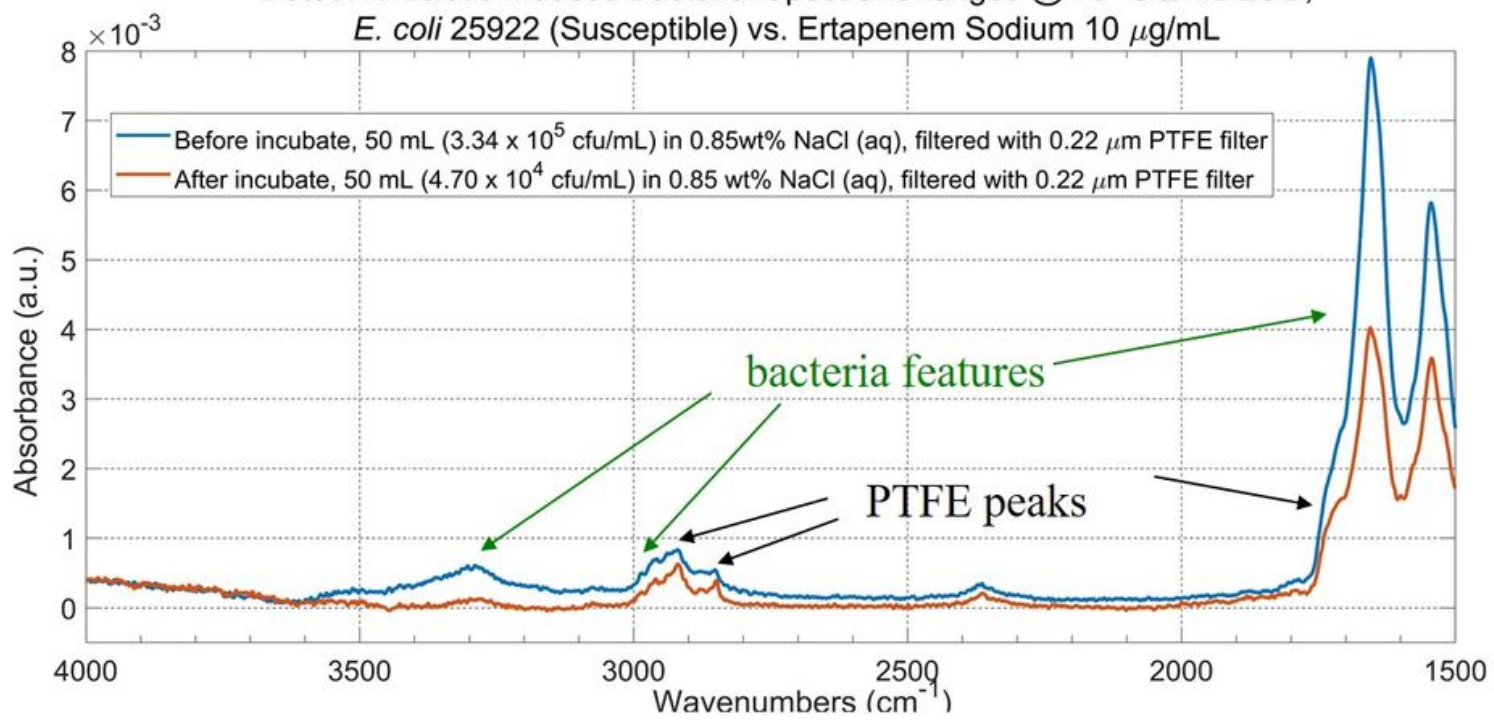

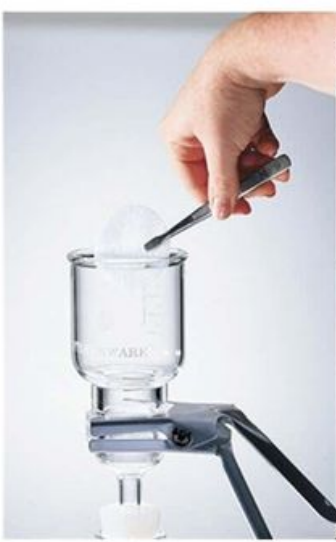

Glass vacuum filtration assembly 
Figure 4

Sensitivity of the ATR system. Antimicrobial-induced bacterial spectral changes can be detected at 104 $\mathrm{cfu} / \mathrm{mL}$ for a E. coli 25922 samples before and after incubation with $10 \mu \mathrm{g} / \mathrm{mL}$ ertapenem sodium at 104 for 4 hours at $35^{\circ} \mathrm{C}$.

(a)

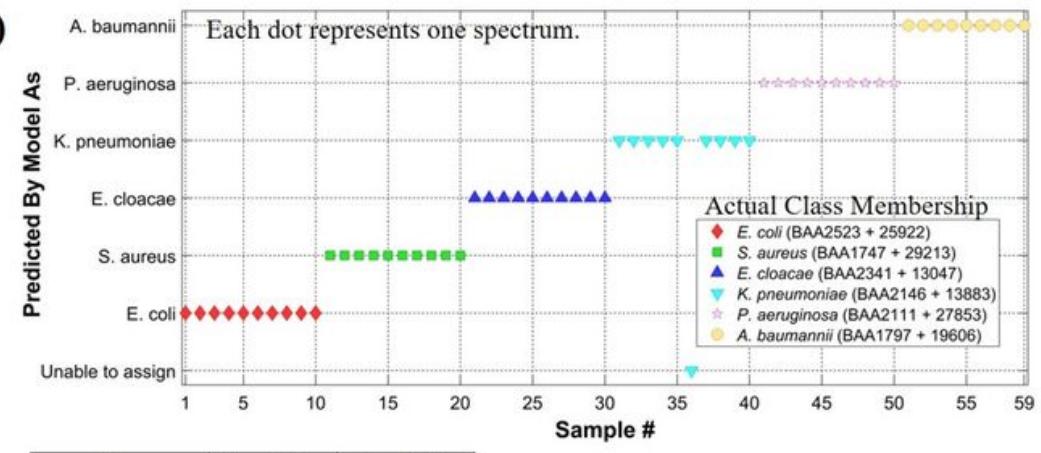

\begin{tabular}{|c|c|c|}
\hline Class & Sensitivity & Specificity \\
\hline E. coli & $100 \%$ & $100 \%$ \\
\hline S. aureus & $100 \%$ & $100 \%$ \\
\hline E. cloacae & $100 \%$ & $100 \%$ \\
\hline K. pneumoniae & $90 \%$ & $100 \%$ \\
\hline P. aeruginosa & $100 \%$ & $100 \%$ \\
\hline A. baumannii & $100 \%$ & $90 \%$ \\
\hline
\end{tabular}

Sensitivity $=$ number of data points predicted as in the class divided by the actual number in the class.

Specificity $=$ number of data points predicted as not in class divided by the actual number of not in the class.

(b)

\begin{tabular}{|c|c|c|c|c|c|c|c|c|}
\hline \multirow[t]{2}{*}{ Example \# } & \multirow[t]{2}{*}{ Actual Class } & \multirow[t]{2}{*}{ \# of sample } & \multicolumn{6}{|c|}{ Predicted as } \\
\hline & & & E. coli & S. aureus & E. cloacae & K. pneumoniae & P. aeruginosa & A. baumannii \\
\hline (a) & E. cloacae BAA2341+8 $\mu \mathrm{g} / \mathrm{mL}$ levofloxacin* & 5 & 0 & 0 & 5 & 0 & 0 & 0 \\
\hline (b) & S. aureus BAA1747 $+20 \mu \mathrm{g} / \mathrm{mL}$ oxacillin sodium* & 3 & 0 & 3 & 0 & 0 & 0 & 0 \\
\hline (c) & E. coli BAA2523 in 1:5 FBS/water & 5 & 5 & 0 & 0 & 0 & 0 & 0 \\
\hline (d) & $\begin{array}{c}\text { K. pneumoniae } 70 \% \text { BAA } 2146+30 \% 13883 \text {, after } \\
8 \mu \mathrm{g} / \mathrm{mL} \text { ceftriaxone disodium salt } \\
\text { hemi(heptahydrate) for } 4 \text { hours @ } 35^{\circ} \mathrm{C}\end{array}$ & 5 & 0 & 0 & 0 & 5 & 0 & 0 \\
\hline
\end{tabular}

\section{Figure 5}

Automatic spectral analysis by machine learning models (a) $>95 \%$ species specificity and sensitivity achieved in distinguishing six bacterial species based on ATR spectral features. (b) numerical summary of classification results by the machine learning model for four different unknown samples.

\section{Supplementary Files}

This is a list of supplementary files associated with this preprint. Click to download.

- SupplimentaryFigures.docx 